\title{
Evaluation of the Separation Mechanism for a Small Carry-on Impactor aboard Hayabusa2
}

\author{
By Takanao SAIKI, ${ }^{1)}$ Hiroshi IMAMURA, ${ }^{1)}$ Naruhisa SANO, ${ }^{2)}$ and Hirohito OHTSUKA ${ }^{2)}$ \\ ${ }^{1)}$ Institute of Space and Astronautical Science, JAXA, Sagamihara, Japan \\ ${ }^{2)}$ IHI AEROSPACE Co. Ltd., Tomioka, Japan
}

(Received March 14th, 2019)

\begin{abstract}
Hayabusa2 is a current sample return mission spacecraft developed by JAXA. One of the most important scientific objectives of Hayabusa2 is to investigate the chemical and physical properties of the internal materials and structures of the asteroid Ryugu. To achieve this goal, we developed a new device called the small carry-on impactor (SCI). The SCI is a kinetic impactor that is being used to create an artificial crater on the surface of Ryugu. It has no attitude or orbit control device. The helical spring in the separation mechanism provides both separation force and spin torque to the SCI. The impact accuracy of the SCI depends heavily on the separation accuracy. Repeating a large number of separation tests is a straightforward approach to evaluate the separation accuracy. However, that is not realistic. Hence, first we performed several element tests to understand the separation dynamics. Then, the final separation accuracy was evaluated through a small number of separation tests, and this was followed by simulations that used the results of the element tests. This paper provides an overview of the separation mechanism, the results of development testing analyzed in detail, and a summary of the separation performance.
\end{abstract}

Key Words: Hayabusa2, Asteroid Exploration, Small Carry-on Impactor, Separation Mechanism

\section{Introduction}

JAXA's current asteroid investigator, Hayabusa2, is now on a mission. It was launched on 3 December 2014 and has arrived at Ryugu, a C-type asteroid. Hayabusa2 is a sample return mission similar to that of Hayabusa. ${ }^{1-3)}$ Hayabusa explored the asteroid Itokawa and enabled many scientific discoveries during its stay on Itokawa through various scientific observations. ${ }^{4-6)}$ Hayabusa determined that Itokawa is a rubblepile body with macroporosity. ${ }^{7)}$ However, no direct observational data on the internal structure or subsurface materials of the asteroid are available. One of the most important scientific objectives of Hayabusa 2 is to investigate the chemical and physical properties of the internal materials and structure of Ryugu. To achieve this goal, Hayabusa 2 is equipped with a new device called the small carry-on impactor (SCI), which is designed to create an artificial crater on the surface of the asteroid. ${ }^{8)}$ The SCI is attached to the bottom panel of the mother spacecraft (MSC). A newly developed separation mechanism for the SCI (SMS) connects the MSC and SCI, and the SCI is separated just before the impact experiment. A particular type of shaped charge in the SCI accelerates the impactor, and the impactor hits the asteroid surface. The final velocity of the impactor is more than $2 \mathrm{~km} / \mathrm{s}$, and its weight is about $2 \mathrm{~kg}$. The Hayabusa 2 spacecraft will attempt to observe the resultant crater with scientific instruments and collect sample materials from the around the artificial crater.

The SCI is a simple kinetic impactor. It must be compact

(C) 2020 The Japan Society for Aeronautical and Space Sciences because the mass and size permitted for the SCI are highly restricted. Accordingly, it has no attitude or orbit control device. The helical spring in the SMS provides the separation force and torque of the SCI, and the spin motion maintains its attitude. As a result, the separation accuracy is a critical factor in achieving a successful impact experiment. Evaluating the separation performance of the SMS is important for the actual impact. Conducting a large number of separation tests would be the best way to assess the separation accuracy. However, this is not realistic due to cost limitations and the development period allowed. Therefore, we adopted the following approach.

First, we performed some element tests to understand the separation dynamics. We investigated the disturbances from the Marman band and the characteristics of the helical spring in element tests. Then, the final performance was confirmed through separation tests using a flight model (FM) of the SMS. The results indicated that the SMS developed satisfied the performance required. However, the number of separations was only six, and we thought that this number was insufficient. Furthermore, the setting of the final separation test was different from the actual configuration. For these reasons, we decided to perform simulations based on the results of the final separation test. The element tests conducted previously enabled us to simulate the motion of separation. We performed a Monte Carlo simulation by changing the disturbances and stiffness of the helical spring, and we found that the required separation accuracy was almost satisfied.

This paper first describes an overview of the SMS, and then the results of the development testing analyzed in detail and the summarized separation performance are shown. 


\section{Small Carry-on Impactor}

\subsection{Outline of SCI}

The SCI is a carry-on-type kinetic impact system aboard the Hayabusa2. Figure 1 shows the SCI before and after separation without thermal blankets. The left-hand-side figure shows the configuration before separation. The main body of the SCI is connected to the SMS using a Marman clamp. The right-hand figure shows the main body after separation. The weight of the main body is about $14 \mathrm{~kg}$, and the weight of the SMS is less than $4 \mathrm{~kg}$.

The SCI consists of three parts. The explosive part has a powerful $4.7 \mathrm{~kg}$ explosive that accelerates the impactor. The electrical part includes a sequencer, an ignition circuit, and primary batteries. The sequencer controls the timing of the impact. The interface part is the separation mechanism. The paper by Saiki et al. ${ }^{8}$ discusses details of the SCI configuration. The impact accuracy depends on the following factors:

1) The attitude error of the MSC at separation,

2) The position and velocity error of the MSC at separation,

3) The attitude error of the SCI,

4) The separation velocity error of the SCI, and

5) The flight direction error of the impact head. We discuss 3) and 4) of the above in this paper.
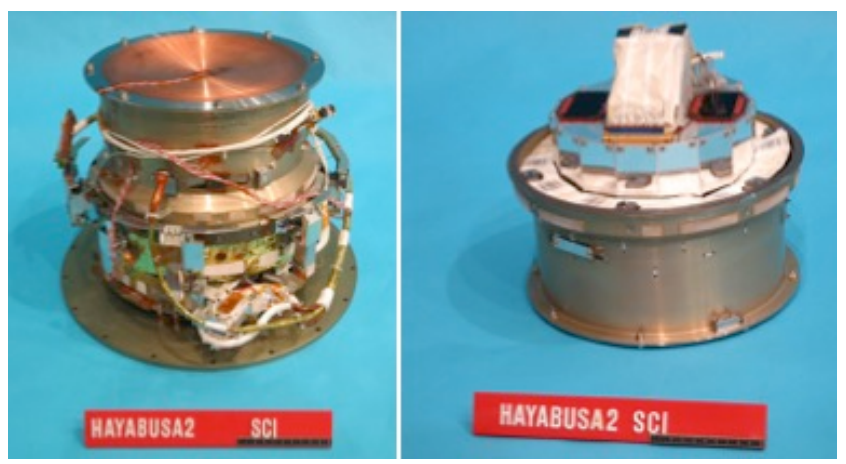

Fig. 1. Hayabusa2 SCI. Left: Configuration before separation. Marman clamps connect the SCI's main body and SMS. Right: Configuration after separation.

\subsection{SCI separation mechanism}

Figure 2 shows the SMS. It includes a helical spring, Marman clamp, separation pullers, and a wire-cutter. Table 1 lists the specifications of the SMS. The SMS has the following functions:

1) Mounting the main body of the SCI to the MSC,

2) Cutting the wire harness before the separation by receiving a signal from the MSC,

3) Releasing the SCI after receiving the separation signal from the MSC, and

4) Giving the separation force and spin torque to the main body of the SCI.

The MSC provides the release signal to the SMS at the time of separation. That signal ignites the separation pullers in the SMS, and the Marman clamps are released.

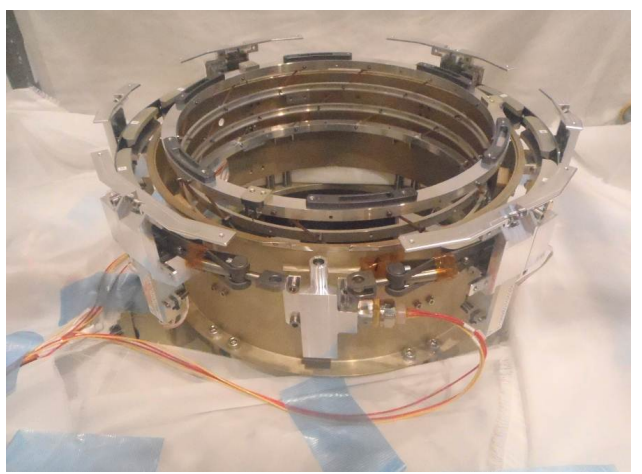

Fig. 2. SCI separation mechanism. A helical spring in it gives spin torque and velocity to the main body of the SCI.

\begin{tabular}{|c|c|}
\hline Items & Values \\
\hline \multirow[t]{5}{*}{ Component } & Structure \\
\hline & Helical spring \\
\hline & Marman clamp \\
\hline & Separation puller (2) \\
\hline & Wire-cutter \\
\hline \multirow[t]{2}{*}{ Size } & Diameter: approx. $300 \mathrm{~mm}$ \\
\hline & Height: approx. $120 \mathrm{~mm}$ \\
\hline Weight & $3.73 \mathrm{~kg}$ \\
\hline Separation & Separation puller is ignited by the MSC \\
\hline Wired $\mathrm{I} / \mathrm{F}$ & Power line $(15 \mathrm{~V})$ \\
\hline *All of the lines are & Communication line (command \& telemetry) \\
\hline cut by the wire-cutter & Temperature monitor line $(8 \mathrm{ch})$ \\
\hline before separation. & Heater power line $(6 \mathrm{ch})$ \\
\hline
\end{tabular}

\subsection{Requirements for separation accuracy}

The attitude and velocity errors of the SCI are critical from the impact accuracy viewpoint. Disturbance force and torque of the SMS at the moment of separation can result in velocity and attitude errors. The leading causes of the disturbance are 1) manufacturing error in the separation spring and 2) disturbance forces from the Marman clamp and separation puller. For the precise impact, the separation errors must be smaller than the maximum errors shown in Table 2.

Table 2. Maximum allowable error.

\begin{tabular}{ll}
\hline Items & Value \\
\hline Separation velocity (axial) & $50 \mathrm{~mm} / \mathrm{s}$ \\
Separation velocity (lateral) & $20 \mathrm{~mm} / \mathrm{s}$ \\
Spin axis pointing error & $20 \mathrm{deg}$ \\
\hline
\end{tabular}

\section{Element Tests during Development Phase}

During the development phase, we conducted the following element tests:

1) Marman clamp release test,

2) Measurement of the helical spring characteristics, and

3) Separation test only using the helical spring.

The primary purpose of the element tests was to evaluate the validity of the design and the manufacturing errors.

The other important objective was to understand the separation dynamics. As the separation motion is quick and complicated, it is difficult to evaluate the separation motion using only one kind of separation test. The element tests were helpful to gain a 
better understanding of the separation motion. The results of the element tests were also useful for simulating the separation process. The following sections show the details and results of the element tests.

\subsection{Marman clamp release test}

Figure 3 shows the Marman clamp of the SMS. Two band clamps connected by lock pins join the main body of the SCI with the SMS. Two explosive pin pullers are used for release. The band catch mechanism in the SMS captures the released band clamps to prevent the released clamps from hitting the devices around the SCI.

We conducted the Marman clamp release test without the helical spring to verify the release mechanism. The primary purposes of the experiment were as follows:

1) Understanding the motion of the band clamps after being released,

2) Verifying that the band catch mechanism works properly, and

3) Evaluation of the shock level at release.

A secondary objective was evaluating the disturbance from the release mechanism. We measured the body rate orthogonal to the spin axis (tip-off rate) in this test. Four laser displacement sensors measured the tip-off rate after releasing the Marman clamp (Fig. 4). The test configuration was almost the same as the configuration shown in Fig. 10. A counterweight canceled the gravity. Table 3 shows the estimated tip-off rate. We estimated the tip-off rate by calculating the approximate line of the laser displacement data for $200 \mathrm{~ms}$ after separation. We found that releasing the Marman clamp can generate a quite large disturbance torque. The results also indicate that variation in the disturbance torque can be quite large.

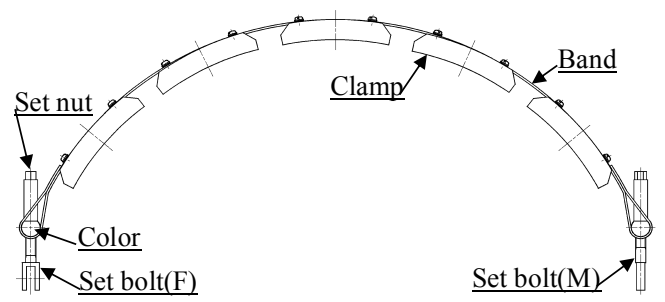

Fig. 3. Marman clamp of the SMS.

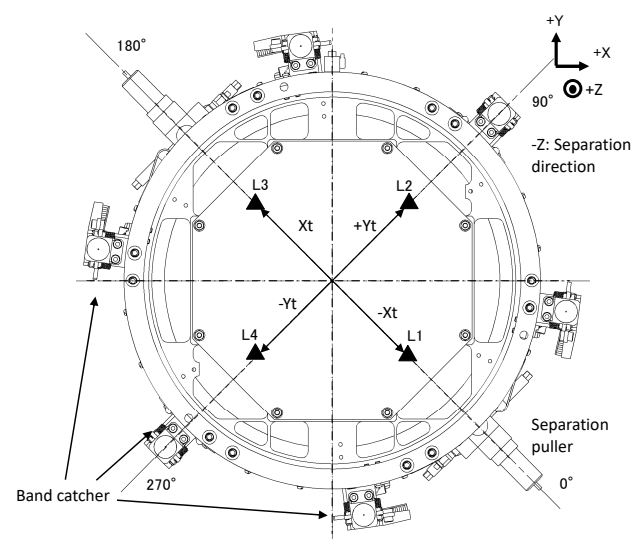

Fig. 4. Measurement points of the laser displacement sensors.
Table 3. Tip-off rate in the Marman clamp release test.

\begin{tabular}{ccc}
\hline No. & $\begin{array}{c}\text { Tension of } \\
\text { band clamp [N] }\end{array}$ & $\begin{array}{c}\text { Tip-off rate } \\
{[\mathrm{deg} / \mathrm{s}]}\end{array}$ \\
\hline 1 & 2513 & 1.5 \\
2 & 2641 & 2.1 \\
3 & 2225 & 2.7 \\
4 & 2596 & 2.7 \\
5 & 2800 & 5.5 \\
6 & 2728 & 5.1 \\
\hline
\end{tabular}

\subsection{Measurement of helical spring characteristics}

Figure 5 shows the helical spring of the SMS. It provides the separation force and spin torque to the SCI's main body. First, we measured the characteristics of the helical spring. Regarding the side force and torque, we derived a mathematical model by assuming the axial symmetry as follows:

$$
\begin{gathered}
\left(\begin{array}{c}
F_{X} \\
F_{Y} \\
M_{X} \\
M_{Y}
\end{array}\right)= \\
{\left[\begin{array}{cccc}
K_{f f} & 0 & K_{f m 1} & -K_{f m 2} \\
0 & K_{f f} & K_{f m 2} & K_{f m 1} \\
K_{f m 1} & K_{f m 2} & K_{m m} & 0 \\
-K_{f m 2} & K_{f m 1} & 0 & K_{m m}
\end{array}\right]\left(\begin{array}{c}
u_{X} \\
u_{y} \\
\theta_{X} \\
\theta_{Y}
\end{array}\right),}
\end{gathered}
$$

where, $F_{X}, F_{Y}$ : restoring side-force, $M_{X}, M_{Y}$ : restoring torque, $u_{X}, u_{Y}$ : lateral displacement, and $\theta_{X}, \theta_{Y}$ : tilt angle. $K_{f f}$ is sharing stiffness and $K_{m m}$ is bending rigidity. $K_{f m 1}$ and $K_{f m 2}$ are the coupling stiffness of the helical spring. Figure 6 shows the definition of side force, torque, and displacement.

First, we investigated the relation between the axial force and the height of the spring. The force was measured using small compression load cells. Figure 7 shows the torque-free axial force of the helical spring. The axial force depends on the height of the spring. The SCI uses the range of $46-70 \mathrm{~mm}$ for separation.

The lateral characteristics were also measured by changing the side force and torque. Figure 8 shows the measurement results. $K_{m m}, K_{f m 1}$, and $K_{f m 2}$ are normalized by $a$, the radius of the spring in this figure. The stiffness is a function of the spring's height.

Measuring the spring's characteristics is very important for understanding separation motion because it enables us to perform the numerical simulations shown in Section 5.

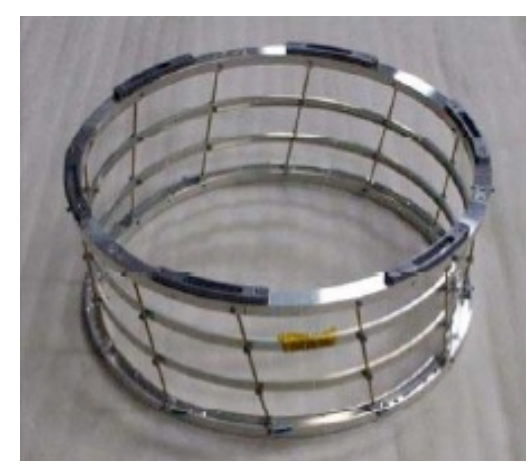

Fig. 5. Helical spring of the SMS. 


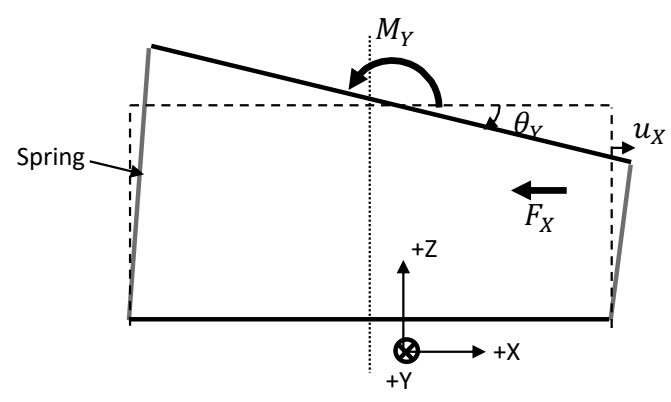

Fig. 6. Definition of the side force, torque, and displacement.

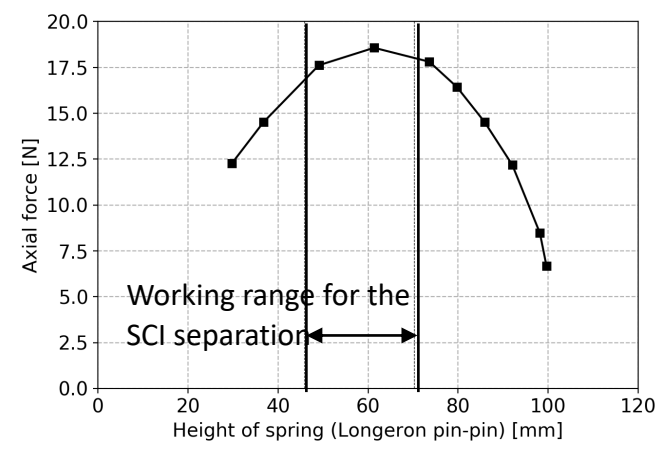

Fig. 7. Axial force of the helical spring.

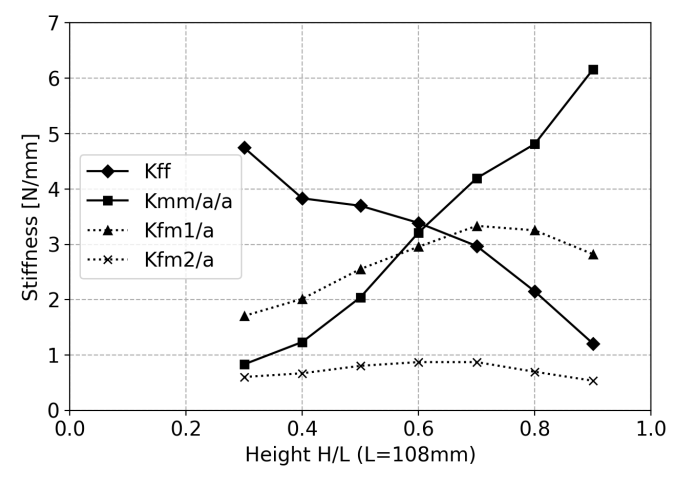

Fig. 8. Helical spring stiffness.

\subsection{Separation test using only helical spring}

After measuring the characteristics of the helical spring, we conducted the separation test using only the helical spring. We found that releasing the Marman clamp was the primary cause of disturbance. Thus, we confirmed the ideal release performance by eliminating the influence of the Marman clamp and pin pullers. Figure 9 shows the test configuration. A rubber tube cancels the gravitational force, and a solenoid statically releases the dummy SCI. Three laser displacement sensors and a high-speed camera observed the motion of the dummy SCI.

The separation velocity and tip-off rate are obtained from the data of the laser displacement sensors. The slope of the averaged data of three displacement sensors gives us the separation velocity. The three displacement sensors also detect the direction of the spin axis. We are able to generate the tilt angle history from the spin axis direction data and estimate the tip-off rate of the dummy SCI. The spin rate and lateral velocity are estimated by analyzing the images of the high-speed camera. The lateral velocity is obtained from the motion of the center of gravity, and the spin rate is estimated by tracking the movement of the edge of the dummy SCI.

Table 4 shows a summary of the separation test results under nominal conditions. The variation in separation velocity is quite small. Both lateral velocity and tip-off rate are also small. This indicates that disturbance from the helical spring itself is quite low.

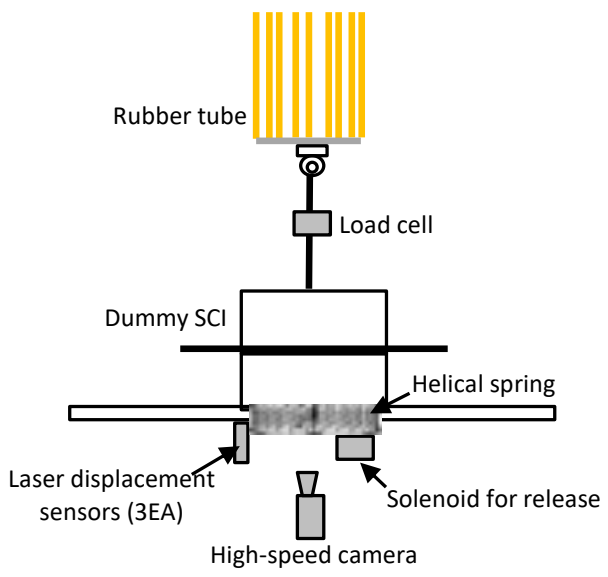

Fig. 9. Configuration of the separation test using only the helical spring. The motion of the dummy SCI is captured by the laser displacement sensors and a high-speed camera.

Table 4. Summary of the separation test (helical spring only, nominal).

\begin{tabular}{ll}
\hline Items & Value \\
\hline Separation velocity (axial) & $215+/-7 \mathrm{~mm} / \mathrm{s}$ \\
Spin rate & $77.9+/-3.1 \mathrm{deg} / \mathrm{s}$ \\
Separation velocity (lateral) & $6.5 \mathrm{~mm} / \mathrm{s}(3 \sigma)$ \\
Tip-off rate & $2.6 \mathrm{deg} / \mathrm{s}(3 \sigma)$ \\
Duration of spring extension & $0.219+/-0.011 \mathrm{sec}$ \\
Separation energy & $0.432+/-0.015 \mathrm{~J}$ \\
\hline
\end{tabular}

\section{Final Integrated Separation Test}

We performed a final separation test for evaluating the SMS. It was a full-configuration test using the FM of the SMS, which includes the helical spring, Marman clamp, and explosive pin pullers; however, a dummy SCI body was used. Figure 10 shows the test configuration. The counterweight canceled the gravity. Two high-speed cameras and four laser displacement sensors observed the motion of the main body when released. The laser sensors measured the axial displacements of the points shown in Fig. 4. The slope of the averaged data of four displacement sensors provided us the separation velocity. The difference between two sensors (L1-L3 and L2-L4) gave us the tip-off rate information. The high-speed cameras observed the motion of the lateral velocity and spin rate by tracking markers mounted on the dummy SCI body.

Table 5 shows the mass and moment of inertia of the dummy SCI. As we used a counterweight in this test, the mass of the dummy SCI is about half that of the FM. This means that we have to convert the test results by considering the difference compared to actual conditions.

Figure 11 shows the axial velocity of the body observed by 
Trans. JSASS Aerospace Tech. Japan Vol. 18, No. 1 (2020)

the laser displacement sensors. It indicates the final separation velocity is about $190 \mathrm{~mm} / \mathrm{s}$ and the duration of spring extension is about $0.2 \mathrm{~s}$. We find the vibration and attenuation using this velocity data. The equation of motion in the axial direction under test conditions can be written as follows:

$$
\left\{\begin{array}{c}
m_{1} \ddot{z}_{1}=-m_{1} g+k_{1} s_{1}+F \\
m_{2} \ddot{z}_{2}=m_{2} g-k_{2} s_{2} \\
I_{1} \ddot{\theta}_{1}=\left(k_{3} s_{3}-k_{1} s_{1}-F_{f}\right) r_{1} \\
I_{2} \ddot{\theta}_{2}=\left(k_{2} s_{2}-k_{3} s_{3}-F_{f}\right) r_{2}, \\
\dot{s}_{1}=-\dot{z}_{1}+r_{1} \dot{\theta}_{1} \\
\dot{s}_{2}=\dot{z}_{2}-r_{2} \dot{\theta}_{2} \\
\dot{s}_{3}=r_{2} \dot{\theta}_{2}-r_{1} \dot{\theta}_{1}
\end{array}\right.
$$

where, the descriptions of the symbols are shown in Fig. 12. Equation (2) indicates that the motion of the body is affected by the wire's rigidity and the friction of the fixed pulleys. Therefore, we have to consider the above forces to precisely estimate the separation velocity.

Figure 13 shows the lateral motion of the main body during and after separation estimated using data from the high-speed cameras and laser sensors. This gives us the vibration response of the body. It indicates that the spring gives the restoring force and torque to the main body during extension.

Table 6 shows a summary of the final separation test results. All of the data are converted while considering the differences between the test conditions and actual conditions. The separation velocity and spin rate were converted such that the estimated kinetic energy of the actual system is equal to the total energy of the test system, including the friction loss. The tip-off rate and lateral velocity were converted by considering the difference in the mass and moment of inertia of the test body between the actual configuration and test configuration. Even when considering three-sigma deviation, we concluded that the estimated accuracy satisfies the requirements shown in Table 2. On the other hand, the number of separations was limited, and the setting for the separation test was quite different from the actual configuration. Therefore, we considered that further analysis was necessary to verify the test results.

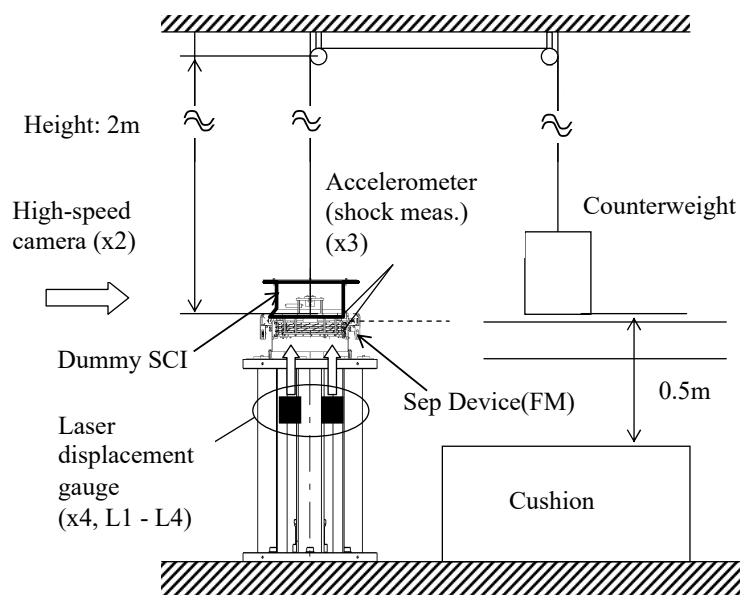

Fig. 10. Configuration of the final separation test. The motion of the separated SCI is monitored using the high-speed cameras and laser displacement sensors.
Table 5. Dummy SCI used in the final separation test.

\begin{tabular}{lcc}
\hline Items & Dummy SCI & FM \\
\hline Mass [kg] & 7.953 & 13.975 \\
& $15.984^{*}$ & \\
Moment of inertia $I_{X}\left[\mathrm{kgm}^{2}\right]$ & 0.118 & 0.121 \\
Moment of inertia $I_{Y}\left[\mathrm{kgm}^{2}\right]$ & 0.118 & 0.117 \\
Moment of inertia $I_{Z}\left[\mathrm{kgm}^{2}\right]$ & 0.129 & 0.126 \\
\hline
\end{tabular}

* Dummy SCI+counterweight+equivalent mass of pulley

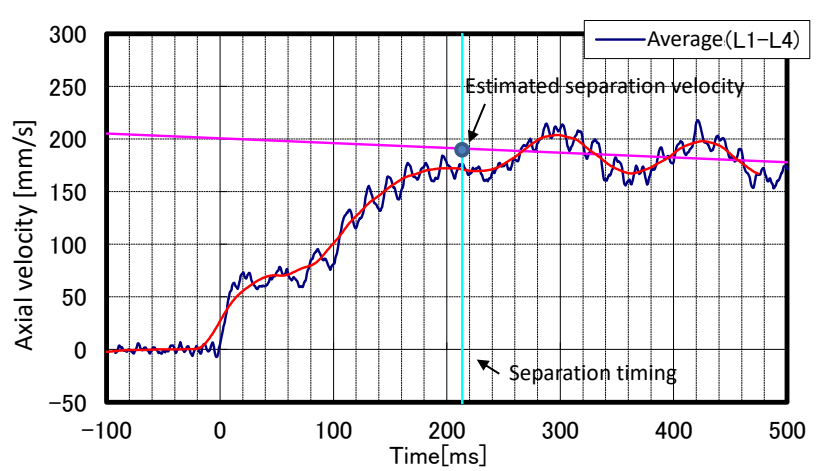

Fig. 11. Velocity history. The velocity was obtained from the data of the laser displacement sensors. The velocity decreases gradually after separation due to the friction.

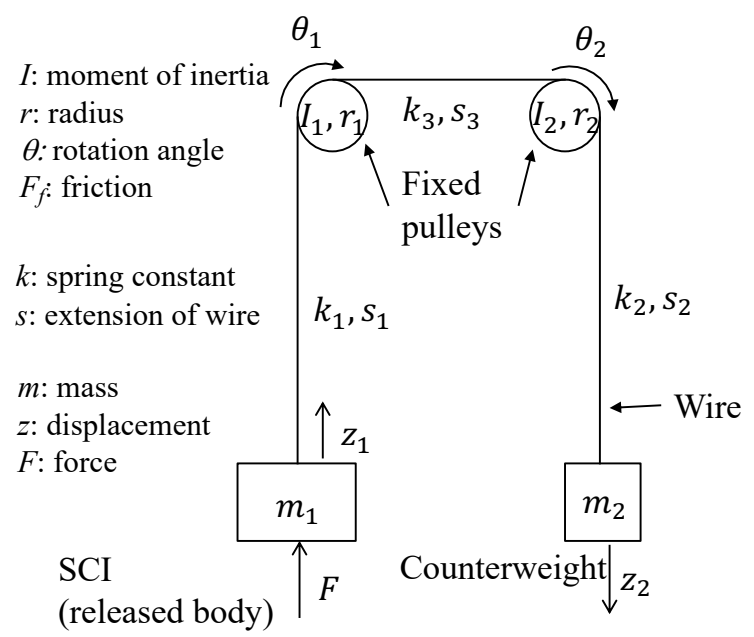

Fig. 12. Motion in the axial direction. A wire rope connects the SCI and a counterweight. We have to consider the wire's rigidity and the friction of the pulleys to precisely estimate the separation velocity.
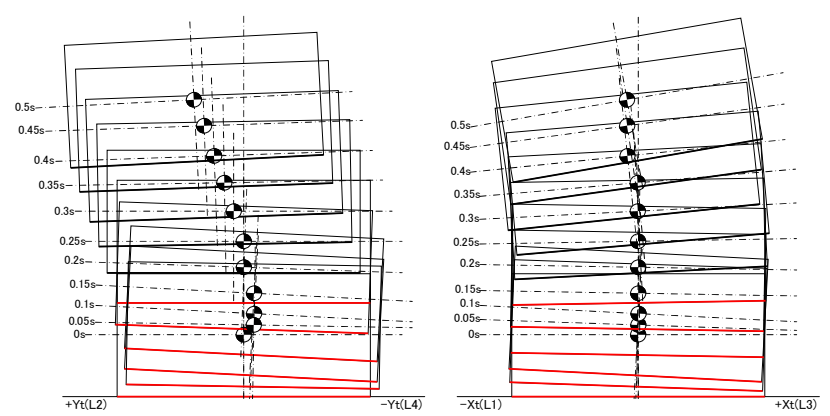

Fig. 13. Lateral motion captured by the high-speed cameras and laser sensors. Xt and Yt in this figure are defined in Fig. 4. 
Trans. JSASS Aerospace Tech. Japan Vol. 18, No. 1 (2020)

Table 6. Results of the final separation test.

\begin{tabular}{lcccc}
\hline & $\begin{array}{c}\text { Separation } \\
\text { vel. [mm/s] }\end{array}$ & $\begin{array}{c}\text { Spin rate } \\
{[\mathrm{deg} / \mathrm{s}]}\end{array}$ & $\begin{array}{c}\text { Lateral } \\
\text { vel. [mm/s] }\end{array}$ & $\begin{array}{c}\text { Tip-off } \\
\text { rate [deg/s] }\end{array}$ \\
\hline FM\#1 & 206 & 74.5 & 9.5 & 3.0 \\
FM\#2 & 213 & 74.0 & 6.4 & 2.9 \\
FM\#3 & 205 & 71.1 & 12.2 & 5.2 \\
FM\#4 & 208 & 75.0 & 7.0 & 2.8 \\
FM\#5 & 209 & 78.2 & 3.5 & 0.9 \\
FM\#6 & 217 & 76.4 & 5.2 & 2.7 \\
Average & 210 & 74.9 & 7.3 & 2.9 \\
$3 \sigma$ & 14 & 7.2 & 9.3 & 4.1 \\
\hline
\end{tabular}

\section{Separation Simulation}

We conducted simulations of the separation motion to verify the results of the final separation test. The axial motion can be written as follows:

$$
\left\{\begin{array}{l}
m \ddot{z}=F_{Z} \\
I_{Z} \ddot{\phi}=M_{Z}
\end{array}\right.
$$

where, $m$ : mass of the SCI, $I_{Z}$ : moment of inertia, $z$ : position, $\phi$ : rotation angle, $F_{Z}$ : axial force, and $M_{Z}$ : spin torque. The energy of the helical spring is distributed to both velocity and spin motion while satisfying the following relationship:

$$
\left\{\begin{array}{c}
F_{T F} d z=F_{Z} d z+M_{Z} d \phi \\
L^{2}=z^{2}+(a \phi)^{2}
\end{array},\right.
$$

where, $F_{T F}$ : axial force (torque-free) shown in Fig. 7, and $L$ : length of the longeron. The lateral motion can be written as follows:

$$
\left\{\begin{array}{c}
m \ddot{x}_{C G}=F_{X} \\
m \ddot{y}_{C G}=F_{Y} \\
I_{X} \ddot{\theta}_{X}=M_{X}+F_{Y} H_{C G} \\
I_{Y} \ddot{\theta}_{Y}=M_{Y}-F_{X} H_{C G} \\
x_{C G}=u_{x}+H_{C G} \theta_{Y} \\
y_{C G}=u_{y}+H_{C G} \theta_{X}
\end{array}\right.
$$

where, $u_{X}, u_{Y}$ : lateral displacement of the spring, $\theta_{X}, \theta_{Y}$ : rotation angle, $x_{C G}, y_{C G}$ :lateral position of CG of the SCI, and $H_{C G}$ : vertical position of $C G$ from the upper surface of the helical spring. $F_{X}, F_{Y}, M_{X}$ and $M_{Y}$ can be calculated using Eq. (1).

Figure 14 shows an example of the simulation results. We give the initial lateral velocity assuming the Marman band disturbance. As this figure shows, the lateral velocity and tipoff rate show vibration behaviors like the separation test results shown in Fig. 13. Additionally, we find that the spring keeps part of the lateral motion energy. This means that not all of the initial disturbance of the velocity and tip-off rate become the final disturbance at separation.

Next, we compared the results of the final separation test with the simulation results. Figure 15 shows the test results (FM \#2) and simulation results. The stiffness data in Fig. 8 are used for the simulation. The simulation results are quite different from the test results. However, the simulation data shows good agreement with the test data after adjusting the stiffness data. Figure 16 shows the simulation output when the stiffness of the spring is doubled. As a result, we assume that stiffness during the separation motion may be different from the stiffness data acquired under static conditions.

Although we repeated six separations in the final separation test, we assumed this was not enough. Additionally, the configuration of the final separation test was different from the actual configuration. Therefore, we performed a Monte Carlo simulation to complement the test results. Table 7 shows the conditions of the simulation. In this simulation, we changed not only the initial disturbance, but also the stiffness of the helical spring while considering the above result. The range of disturbance was derived from the results of the separation test. Figure 17 shows the simulation results, and the summary is shown in Table 8 . They indicate that the simulation results are almost the same as the final separation test results. We also found that the separation accuracy nearly satisfies the requirement in Table 2, even if we consider a wide range of stiffness.

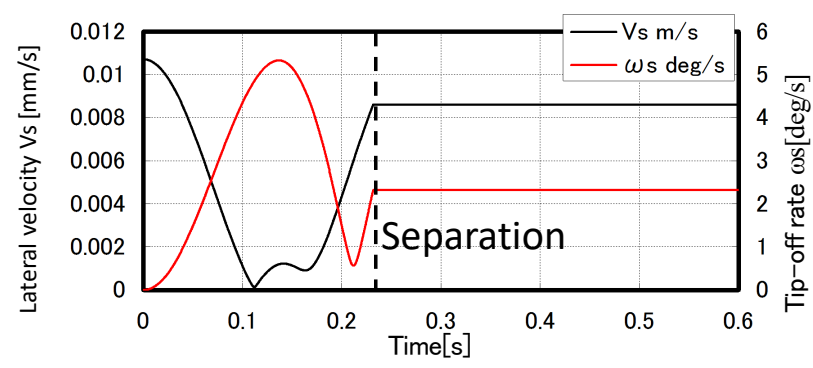

Fig. 14. An example of separation results. The lateral motion shows vibration behavior.

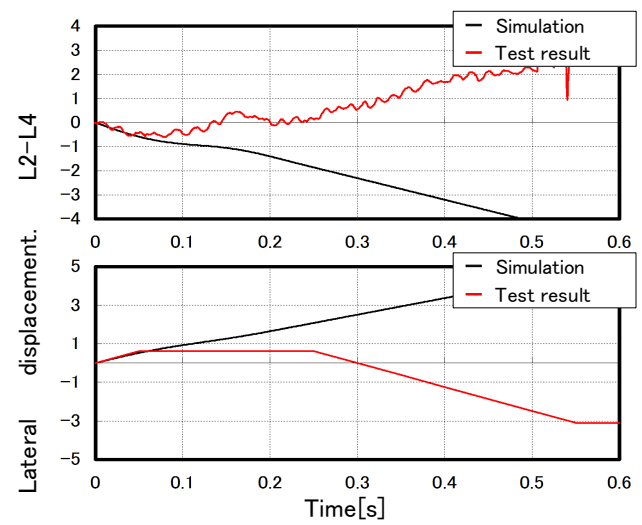

Fig. 15. Results of the separation test and simulation. The stiffness of the spring in Fig. 6 is considered. Upper: Data from the laser displacement sensor. Lower: Lateral displacement data.

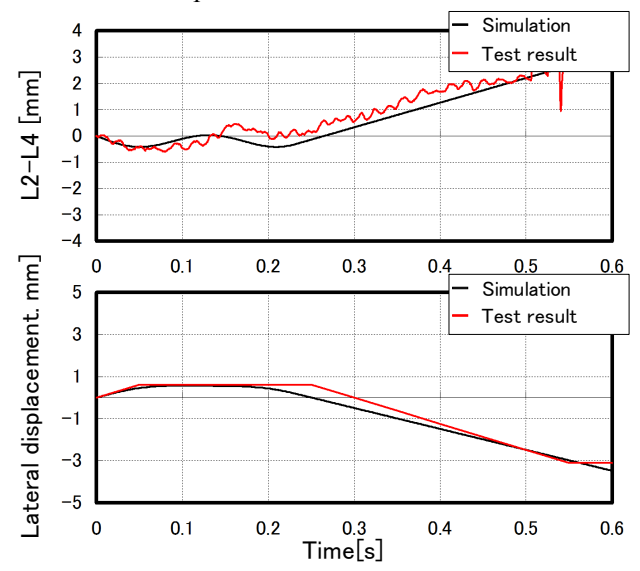

Fig. 16. Simulation results considering the doubled stiffness of the helical spring. 
Table 7. Conditions of the Monte Carlo simulation.

\begin{tabular}{ll}
\hline Items & Value \\
\hline Deviation in axial force & $+/-10 \%$ \\
Spring stiffness & $80-250 \%$ \\
Initial lateral velocity & $10.3 \mathrm{~mm} / \mathrm{s}$ (average) \\
(disturbance) & $8.8 \mathrm{~mm} / \mathrm{s}(3 \sigma)$ \\
Initial tip-off rate & $4.3 \mathrm{deg} / \mathrm{s}($ average) \\
(disturbance) & $6.3 \mathrm{deg} / \mathrm{s}(3 \sigma)$ \\
\hline
\end{tabular}
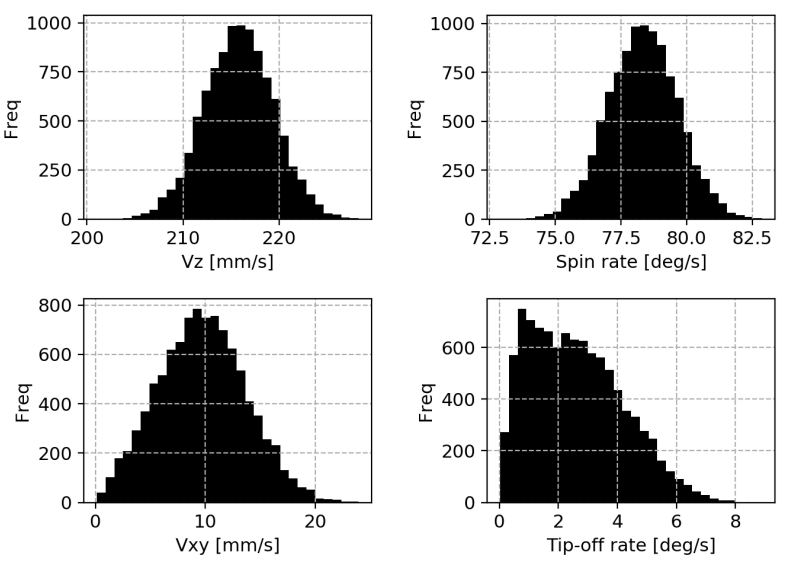

Fig. 17. Results of the Monte Carlo simulation.

Table 8. Summary of the Monte Carlo simulation.

\begin{tabular}{ll}
\hline Items & Value \\
\hline Separation velocity (axial) & $216 \mathrm{~mm} / \mathrm{s}$ (average) \\
& $10.9 \mathrm{~mm} / \mathrm{s}(3 \sigma)$ \\
Spin rate & $78.3 \mathrm{deg} / \mathrm{s}$ (average) \\
& $3.9 \mathrm{deg} / \mathrm{s}(3 \sigma)$ \\
Separation velocity (lateral) & $9.7 \mathrm{~mm} / \mathrm{s}($ average $)$ \\
& $11.9 \mathrm{~mm} / \mathrm{s}(3 \sigma)$ \\
Tip-off rate & $2.6 \mathrm{deg} / \mathrm{s}($ average $)$ \\
& $4.7 \mathrm{deg} / \mathrm{s}(3 \sigma)$ \\
\hline
\end{tabular}

\section{Spring Check before Actual Operation}

We conducted a final spring check in February 2019. The purpose of the check was to confirm the creep effect. We have kept the engineering model (EM) of the helical spring in the compressed condition since the launch of the spacecraft. We measured the axial force and compared it with the axial force data before the launch shown in Fig. 7. We found the energy of the spring had decreased by $3 \%$ and we concluded that the reduction will have little impact on the separation velocity.

\section{Conclusion}

This paper describes the overview of the separation mechanism of the SCI and related separation performance. The separation accuracy of the SMS is a critical factor for the success of the impact mission of Hayabusa2. Understanding the separation motion and dynamics is very important to evaluate the separation accuracy, because it is not possible to repeat a large number of separation tests. Therefore, we conducted several kinds of element tests to deepen our understanding of the separation dynamics. We found that the primary source of disturbance was at the time of releasing the Marman clamp and that separation accuracy without the Marman clamp was quite good. We also obtained the helical spring characteristics. Finally, we were able to confirm separation accuracy during the final separation test using a full configuration, and the results were satisfactory. The results of the final separation test were verified using Monte Carlo simulation. In the simulation, the stiffness data obtained during element tests was used. The results of the simulation and test were in good agreement, even if we consider the uncertainty of the spring's stiffness. We conducted a final spring check in February 2019 and all SMS confirmations were finished. The impact operation of Hayabusa 2 is scheduled to be conducted around the spring of 2019.

\section{References}

1) Yoshikawa, M., and Hayabusa2 Project Team: Outline of the Next Asteroid Sample Return Mission of Japan - Hayaubsa-2, 28th International Symposium on Space Technology and Science, Okinawa, Japan, 2011-k-19, 2011.

2) Tsuda, Y., Yoshikawa, M., Abe, M., Minamino, M., and Nakazawa, S.: System Design of the Hayabusa2 - Asteroid Sample Return Mission to 1999 JU3, Acta Astronautica, 91 (2013), pp. 356-362.

3) Yoshikawa, M., Watanabe, S., Tsuda, Y., Kuninaka, H., and Hayabusa2 project team: Hayabusa2 - the Next Asteroid Sample Return Mission of Japan, Trans. JSASS Aerosp. Technol. Jpn., 12, ists29 (2014), pp. Tk_29-Tk_33.

4) Abe, M., Takagi, Y., Y. Kitazato, K., Abe, S., Hiroi, T., Vilas, F., Clark, B.E., Abell, P.A., Lederer, S.M., Jarvis, K.S., Nimura, T., Ueda, Y., Fujiwara, A.: Near-infrared Ppectral Results of Asteroid Itokawa from the Hayabusa Spacecraft. Science, 312 (2006), 13341338.

5) Saito, J., Miyamoto, H., Nakamura, R., Ishiguro, M., Michikami, T., Nakamura, A. M., Demura, H., Sasaki, S., Hirata, N., Honda, C., Yamamoto, A., Yokota, Y., Fuse, T., Yoshida, F., Tholen, D. J., Gaskell, R. W., Hashimoto, T., Kubota, T., Higuchi, Y., Nakamura, T., Smith, P., Hiraoka, K., Honda, T., Kobayashi, S., Furuya, M., Matsumoto, N., Nemoto, E., Yukishita, K., Kitazato, K., Dermawan, B., Sogame, A., Terazono, J., Shinohara, C., Akiyama, H.: Detailed Images of Asteroid 25143 Itokawa from Hayabusa. Science, 312 (2006), pp. 1341-1344.

6) Yano, H., Kubota, T., Miyamoto, H., Okada, T., Scheeres, D., Takagi, Y., Yoshida, M., Abe, M., Abe, S., Barnouin-Jha, O., Fujiwara, A., Hasegawa, S., Hashimoto, T., Ishiguro, M., Kato, M., Kawaguchi, J., Mukai, T., Saito, J., Sasaki, S., Yoshikawa, M.: Touchdown of the Hayabusa Spacecraft at the Muses Sea on Itokawa. Science, 312 (2006), pp. 1350-1353.

7) Fujiwara, A., Kawaguchi, J., Yeomans, D.K., Abe, M., Mukai, T., Okada, T., Saito, J., Yano, H., Yoshikawa, M., Scheeres, D. J., Barnouin-Jha, O., Cheng, A. F., Demura, H., Gaskell, R. W., Hirata, N., Ikeda, H., Kominato, T., Miyamoto, H., Nakamura, A. M, Nakamura, R., Sasaki, S., Uesugi, K.: The Rubble-pile Asteroid Itokawa as Observed by Hayabusa. Science, 312 (2006), pp. 13301334.

8) Saiki, T., Imamura, H., Aarakawa, M., Wada, K., Takagi, Y., Hayakawa, M., Shirai, K., Yano, H., and Okamoto, C.: The Small Carry-on Impactor (SCI) and the Hayabusa2 Impact Experiment, Space Sci. Rev., 208 (2017), pp. 165-186. 